\title{
A MACROSCOPIC TRAFFIC MODEL FOR ROAD NETWORKS WITH A REPRESENTATION OF THE CAPACITY DROP PHENOMENON AT THE JUNCTIONS
}

\author{
B. Haut ${ }^{*}$ G. Bastin ${ }^{* *}$ Y. Chitour ${ }^{* * *}$ \\ * Aspirant FNRS, haut@auto.ucl.ac.be, Centre for Systems \\ Engineering and Applied Mechanics (CESAME), \\ Université Catholique de Louvain, Bâtiment Euler, 4-6, \\ Avenue Georges Lemaître, 1348 Louvain-la-Neuve, \\ Belgium \\ **bastin@auto.ucl.ac.be, CESAME \\ ***Yacine.Chitour@math.u-psud.fr, Département de \\ Mathématiques, Université Paris-Sud
}

\begin{abstract}
This article deals with the modelling of a road network from a macroscopic point of view. First, the existing models for a road network based on the LWR model are reviewed. Then, these models are extended to take account of the capacity drop phenomenon. The consequences of this modification are finally illustrated in a particular case. Copyright ${ }^{\circledR} 2005$ IFAC
\end{abstract}

Keywords: LWR traffic model, Road network, Junction, Capacity drop, systems of laws of conservation

\section{INTRODUCTION}

A good representation of the evolution of the traffic state on a road network is necessary for the analysis of congestion control strategies. The $c a$ pacity drop phenomenon is a critical phenomenon which represents the fact that the outflow of a traffic jam is significantly lower than the maximum achievable flow at the same location. We can easily understand this phenomenon at a junction where two roads merge in one : if there are too many vehicles trying to access the same road, there is a sort of mutual embarrassment between the drivers which results in an outgoing flow lower than the optimal possible flow.

\footnotetext{
1 This paper presents research results of the Belgian Programme on Interuniversity Attraction Poles, initiated by the Belgian Federal Science Policy Office. The scientific responsibility rests with its author(s).
}

This phenomenon has been experimentally observed (see Cassidy and Bertini (1999) and Hall and Agyemang-Duah (1991)). The flow decrease, which may range up to $15 \%$, has a considerable influence when considering traffic control (Papageorgiou (2002)). Having a model describing this phenomenon is thus a critical element in the establishment of a traffic state regulation strategy.

There exists already some models, like the METANET cell-based model (see Papageorgiou et al. (1990)), which are able to reproduce some of the interesting behaviours on a network like the capacity drop phenomenon at the end of a traffic jam. In the case of macroscopic time and space continuous models, there exist various network models like the models developed by Holden and Risebro (Holden and Risebro (1995)), by Coclite and Piccoli (Coclite et al. (2004)) and by Herty and Klar (Herty and Klar (2003)). But none 
of these previous macroscopic models are able to represent the capacity drop phenomenon. In this article, we will present an extension of these models in order to represent this phenomenon and the important consequences of this modification on the traffic behaviour. The main consequences of this modification are the larger amplitude of the traffic jams occurring at junctions and also that some traffic jams, which were previously transient, become permanent.

Recently, Lebacque (see Lebacque (2004)) has proposed a model able to represent this drop phenomenon by introducing a new state variable describing the state of the junction. In this article, we will develop such model without the introduction of new state variables. We will develop our model on the basis of the LighthillWhitham-Richards (LWR) model (see Lighthill and Whitham (1955) and Richards (1956)) for the description of the traffic state evolution on a single road. In Section 2, we review the existing network models based on the LWR model. In Section 3, a new model of the junctions is introduced which incorporates a representation of the capacity drop phenomenon. The consequences will be presented in Section 4.

\section{THE LWR ROAD NETWORK MODELS}

\subsection{The LWR single road model}

In LWR models, the traffic state is represented from a macroscopic point of view by the function $\rho(x, t)$ which represents the density of vehicles at position $x$ and time $t$. The dynamics of the traffic are represented by a conservation law expressed as

$$
\frac{\partial \rho}{\partial t}+\frac{\partial(\rho v)}{\partial x}=0
$$

where $v=v(x, t)$ is the velocity of cars at $(x, t)$. The main assumption of the LWR model is that the drivers instantaneously adapt their speed in function of the surrounding density i.e. :

$$
v(x, t)=V(\rho(x, t)) .
$$

The function $f(\rho)=\rho V(\rho)$ is then the "flow rate" representing the number of vehicles per time unit passing through a particular position in function of the traffic state at this position. Inserting (2) in (1), the LWR model is :

$$
\frac{\partial \rho}{\partial t}+\frac{\partial f(\rho)}{\partial x}=0
$$

In accordance with the physical observations, it is usually assumed that the speed-density relation is a decreasing function $\left(\frac{\partial V}{\partial \rho}<0\right)$ defined on the interval $\left[0, \rho_{\max }\right]$ with :

$V(0)=V_{\max }:$ the maximal velocity of the vehicles when the road is (almost) empty;
$V\left(\rho_{\max }\right)=0$ : the velocity drops to zero when the density is maximal and the traffic is totally congested.

Then the flow rate $f(\rho)=\rho V(\rho)$ is a non monotonic function with $f(0)=0$ and $f\left(\rho_{\max }\right)=0$ which is maximal at some critical value $\sigma$ : the traffic is moving freely when $\rho<\sigma$ while the traffic is congested when $\rho>\sigma$ (see Fig. 1).

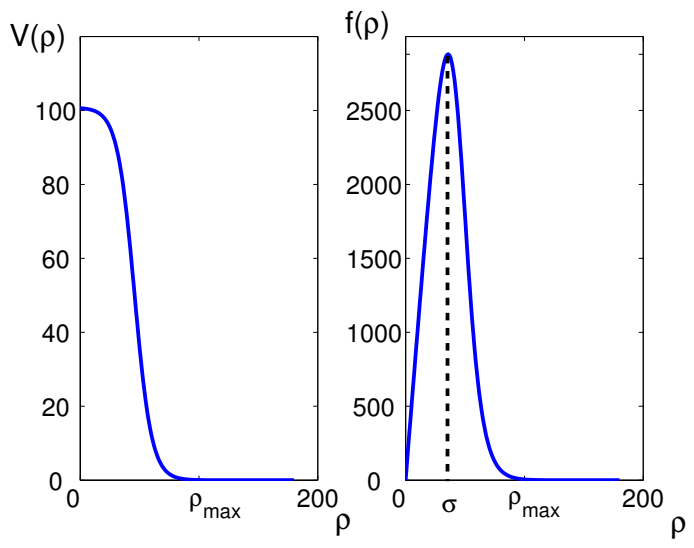

Fig. 1. The speed and the flow in function of the density.

A traditional problem studied for a conservation law of the form (3) is the Riemann problem which is an initial value problem where the initial condition consists of two constant values :

$$
\rho(x, 0)=\left\{\begin{array}{lll}
\rho_{l} & \text { if } & x \leq 0 \\
\rho_{r} & \text { if } & x>0 .
\end{array}\right.
$$

If the function $f(\rho)$ is concave, the solution of the Riemann problem consists in the connexion of the initial states $\left(\rho_{l}\right.$ and $\left.\rho_{r}\right)$ by a wave of one of the following types :

- shock wave if $\rho_{r}>\rho_{l}$. This shock wave (a discontinuity in $\rho$ ) is moving at the speed $\frac{f\left(\rho_{r}\right)-f\left(\rho_{l}\right)}{\rho_{r}-\rho_{l}}$;

- rarefaction wave if $\rho_{r}<\rho_{l}$. The rarefaction is a self-similar solution, i.e. it depends only on $x / t$. The space occupied by the rarefaction wave at time $t$ is $\left[f^{\prime}\left(\rho_{l}\right) t, f^{\prime}\left(\rho_{r}\right) t\right]$.

The Riemann problem is important, not only because it allows an explicit solution but also because the solution of any initial value problem with arbitrary initial conditions can be constructed from a set of appropriate Riemann problems (see e.g. Bressan (2000)).

However, the idea presented in this article doesn't rely on the concave assumption, a non-concave function $f(\rho)$ as represented in Figure 1 may be used as well for the simulations.

\subsection{The network model}

The LWR model (3) is defined for a single unidirectional road. But in this paper, we are concerned 
with the analysis of traffic congestion in road networks.

The simplest network we consider is a junction composed of two incoming and one outgoing infinite roads (see Fig. 2). Naturally, we assume that the density on each link satisfies the single road LWR model

$\frac{\partial \rho_{i}}{\partial t}+\frac{\partial f\left(\rho_{i}\right)}{\partial x_{i}}=0 \quad \forall t, x_{i} \in \begin{cases}]-\infty, 0[ & i=1,2 \\ ] 0, \infty[ & i=3 .\end{cases}$

In order to complete the model, we need to describe the mechanism that occurs at the junction. A first condition is the conservation of flows

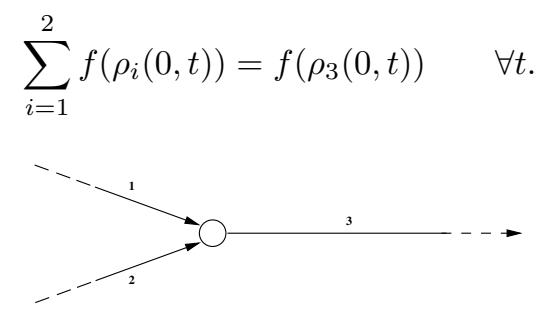

Fig. 2. A simple network.

Like for the single road model, one of the elementary problem we can study, and from which a global solution will be constructed, is the Riemann problem. For a Riemann problem at a junction, we take as initial condition a constant density on the three roads :

$$
\rho_{i}\left(x_{i}, 0\right)=\rho_{i, 0} \quad i=1,2,3 .
$$

However as it can be expected, for the system of conservation laws (4), the boundary condition (5) and the initial condition (6) are not sufficient to have a unique solution to the Riemann problem at the junction. In addition, some of the admissible solutions may be totally unrealistic. For instance, $\bar{\rho}_{1}=\bar{\rho}_{2}=\rho_{\max }, \bar{\rho}_{3}=0$ is always a possible mathematical solution although is is clearly counterintuitive (except obviously in the presence of a red light at the entrance of the third road). A natural way to have a unique solution is to add a model describing the behaviour of the drivers at the junction.

\subsection{A brief review of some junction models}

To describe a solution of a Riemann problem at a junction, given the initial condition (6) at time $t$, the model must express the values of the new densities at the borders of the junction at time $t^{+}$:

$$
\begin{aligned}
& \rho_{i}\left(0, t^{+}\right)=\bar{\rho}_{i} \quad i=1,2 \\
& \rho_{3}\left(0, t^{+}\right)=\bar{\rho}_{3} .
\end{aligned}
$$

After having specified the values of the densities at the borders of the roads, the solution of the
Riemann problem at the junction will be based on the Riemann problems on the different roads :

$$
\begin{aligned}
& \left\{\begin{array}{l}
\rho_{i}\left(x_{i}, 0\right)=\rho_{i, 0} \quad \forall x_{i}<0 \quad i=1,2 \\
\rho_{i}(0,0)=\bar{\rho}_{i}
\end{array}\right. \\
& \left\{\begin{array}{l}
\rho_{3}(0,0)=\bar{\rho}_{3} \\
\rho_{3}\left(x_{3}, 0\right)=\rho_{3,0} \quad \forall x_{3}>0 .
\end{array}\right.
\end{aligned}
$$

Several models of junctions have been proposed in the literature (see Coclite et al. (2004), Herty and Klar (2003), Jin and Zhang (2003), Lebacque and Khoshyaran (1998-2002) and Holden and Risebro (1995)). They all involve the following condition :

Because the wave $\left(\rho_{i, 0}-\bar{\rho}_{i}\right)$ produced on an incoming road by the Riemann problem at the junction must have a negative speed (to not immediately re-enter in the junction), the possible values for $\bar{\rho}_{i}$ must be restricted to a subset of $\left[0, \rho_{\max }\right]$. This is the same for the outgoing road where the speed of the waves $\left(\bar{\rho}_{3}-\rho_{3,0}\right)$ produced by the Riemann problem at the junction must be positive. Based on the speed of the waves described in section 2.1 , one can show that we must have

$$
\begin{aligned}
& \bar{\rho}_{i} \in \begin{cases}\left.\left.\left\{\rho_{i, 0}\right\} \cup\right] \tau\left(\rho_{i, 0}\right), \rho_{\max }\right] & \text { if } 0 \leq \rho_{i, 0}<\sigma \\
{\left[\sigma, \rho_{\max }\right]} & \text { if } \sigma \leq \rho_{i, 0} \leq \rho_{\max }\end{cases} \\
& i=1,2 \\
& \bar{\rho}_{i} \in \begin{cases}{[0, \sigma]} & \text { if } 0 \leq \rho_{i, 0} \leq \sigma \\
\left\{\rho_{i, 0}\right\} \cup\left[0, \tau\left(\rho_{i, 0}\right)[\right. & \text { if } \sigma<\rho_{i, 0} \leq \rho_{\max }\end{cases} \\
& i=3
\end{aligned}
$$

where for each $\rho \neq \sigma, \tau(\rho)$ is the unique number $\tau(\rho) \neq \rho$ such that $f(\rho)=f(\tau(\rho))$. In terms of flow, the admissible regions for the flows are

$$
\begin{gathered}
f\left(\bar{\rho}_{i}\right) \in\left[0, S_{i}\right]= \begin{cases}{\left[0, f\left(\rho_{i, 0}\right)\right]} & \text { if } 0 \leq \rho_{i, 0} \leq \sigma \\
{[0, f(\sigma)]} & \text { if } \sigma \leq \rho_{i, 0} \leq \rho_{\max } \\
& i=1,2\end{cases} \\
f\left(\bar{\rho}_{i}\right) \in\left[0, R_{i}\right]= \begin{cases}{[0, f(\sigma)]} & \text { if } 0 \leq \rho_{i, 0} \leq \sigma \\
{\left[0, f\left(\rho_{i, 0}\right)\right]} & \text { if } \sigma \leq \rho_{i, 0} \leq \rho_{\max } \\
i=3\end{cases}
\end{gathered}
$$

where $S_{i}$ represents the "sending" capacity (sometimes called the traffic demand) of an incoming road and $R_{i}$ the "receiving" capacity (the traffic supply) of an outgoing road (see Daganzo (1995) and Lebacque (2004)).

In our simple network represented in Fig. 2, a natural additional condition to (5) and (7) could be the maximisation of the passing flow :

$$
\sum_{i=1}^{2} f\left(\bar{\rho}_{i}\right) \text {. }
$$

In the case where $S_{1}+S_{2} \leq R_{3}$, the maximum is unique

$$
\left\{\begin{array}{l}
f\left(\bar{\rho}_{i}\right)=S_{i} \\
f\left(\bar{\rho}_{3}\right)=S_{1}+S_{2}
\end{array} \quad i=1,2\right.
$$


If $S_{1}+S_{2}>R_{3}$, we must give some "priority factors" between the incoming flows. Several are possible (see Jin and Zhang (2003)) :

- the priority factors may be function of the incoming flows

$$
\alpha_{i}=\frac{S_{i}}{\sum_{i=1}^{2} S_{i}} ;
$$

- the priority factors may depend on some fixed coefficients $p_{i}$ depending on the road geometry

$$
\alpha_{i}= \begin{cases}p_{i} & R_{3} p_{i} \leq S_{i} \\ \frac{S_{i}}{R_{3}} & S_{i}<R_{3} p_{i}\end{cases}
$$

The possible passing flow is then split between the incoming roads in function of these priority factors :

$$
\left\{\begin{array}{l}
f\left(\bar{\rho}_{i}\right)=\alpha_{i} R_{3} \quad i=1,2 \\
f\left(\bar{\rho}_{3}\right)=R_{3} .
\end{array}\right.
$$

In the case of multiple incoming-outgoing roads, some other models were developed (see Holden and Risebro (1995) and Coclite et al. (2004)).

\section{A NEW MODEL FOR THE JUNCTIONS}

As we have already said in the introduction, the capacity drop phenomenon has a significant importance when considering the establishment of a regulation strategy. However, none of the previous cited models are able to represent this capacity drop phenomenon.

Instead of maximising the criterion over the region defined by (7), we suggest to make the maximisation over a subregion. Defining

$$
R_{3}^{\prime}=\min \left(R_{3}, g\left(\sum_{i} S_{i}\right)\right)
$$

where $g(x)$ is a function whose shape is represented in Figure 3.

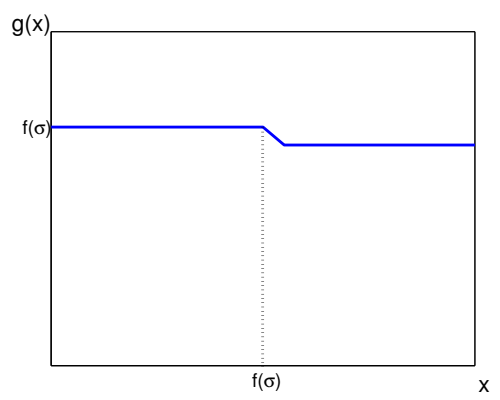

Fig. 3. Possible shape of a $g$ - "capacity drop function".

In this expression of $R_{3}^{\prime}$, we have

- $\sum_{i} S_{i}$ which represents the sum of the flows wishing to enter the outgoing road;
- the function $g(\cdot)$ which expresses the fact that when too many vehicles are trying to enter in the same road $\left(\sum_{i} S_{i}>f(\sigma)\right)$, there is a sort of mutual embarrassment which decreases the capacity of the outgoing road $\left(R_{3}^{\prime} \leq g(\cdot)<f(\sigma)\right)$

- the $\min \left(R_{3}, \cdot\right)$ to be guarantee to remain in a subregion of (7).

We may now optimise any of the criteria mentioned in section 2.3 with

$$
f\left(\bar{\rho}_{i}\right) \in\left\{\begin{array}{l}
{\left[0, S_{i}\right] \quad i=1,2} \\
{\left[0, R_{3}^{\prime}\right] \quad i=3}
\end{array}\right.
$$

instead of (7).

This redefinition of the reception capacity is a correct expression of the capacity drop phenomenon : if too many drivers are trying to access the same road (a traffic jam is occurring), the output flow will be lower than the maximum achievable flow $(f(\sigma))$. As we will see in the next section, this modification has significant consequences on the system behaviour.

\section{SIMULATION EXPERIMENTS}

In this section, the consequences of the capacity drop at the junctions will be presented for the simple network represented in Figure 2. The function $f(\rho)$ used is represented in Figure 1 with $f(\sigma)=2880[\operatorname{veh} / \mathrm{h}]$.

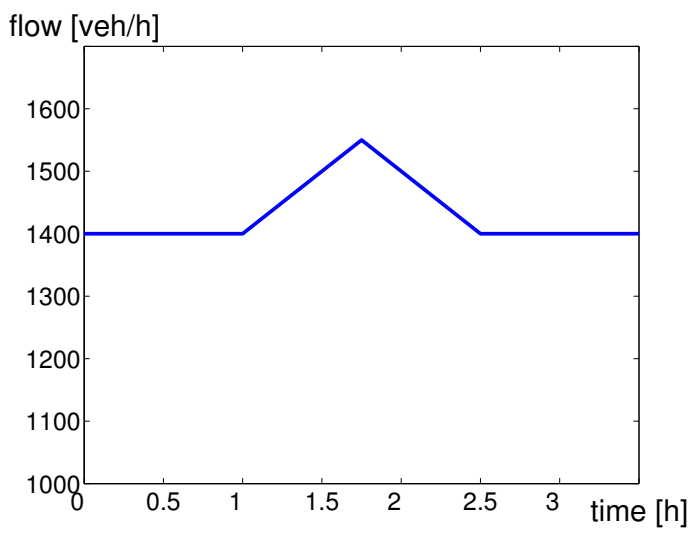

Fig. 4. The demand at the entrance of the first incoming road.

As initial condition, we take 1400 [veh/h] for the flows on the two incoming roads and 2800 on the outgoing one. This is an admissible state for (7) and it is optimal for all the criteria presented in section 2.3. After a while, a temporary increase of flow up to 1550 is added at the beginning of the first incoming road (see Fig. 4). The sum of the two incoming flows $(1400+1550=2950)$ is too high for the outgoing road so a traffic jam will occur. We may now distinguish two cases : 
without the capacity drop representation : the traffic jam will grow and, when the incoming flow on the first road will finally go back to 1400 , the traffic jam will decrease to finally disappear (see Fig. 5 where the traffic state on road 1 and road 2 are represented).

with the capacity drop representation : because of the presence of the traffic jams in front of the junction, the reception capacity of the outgoing road will drop down to 2736 $(=0.95 f(\sigma))$. The traffic jam will grow and, even after that the incoming flow on the first road has gone back to 1400 , the sum of the incoming flows is too high for the new reception capacity of the outgoing road $(1400+1400>$ 2736). As we can see in Fig. 6, the traffic jam will never stop of growing. In order to make the jam disappear, the incoming flow must be significantly lower than it was before the creation of the jam.
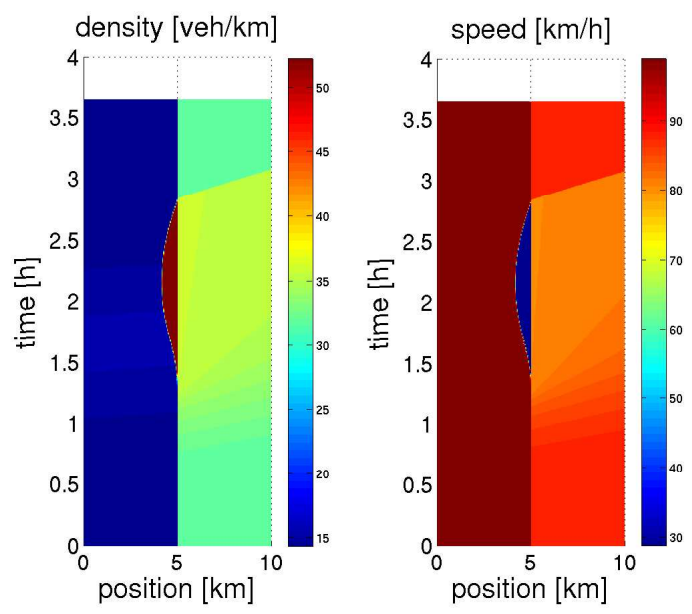

Fig. 5. The evolution of the traffic state in absence of a capacity drop representation.
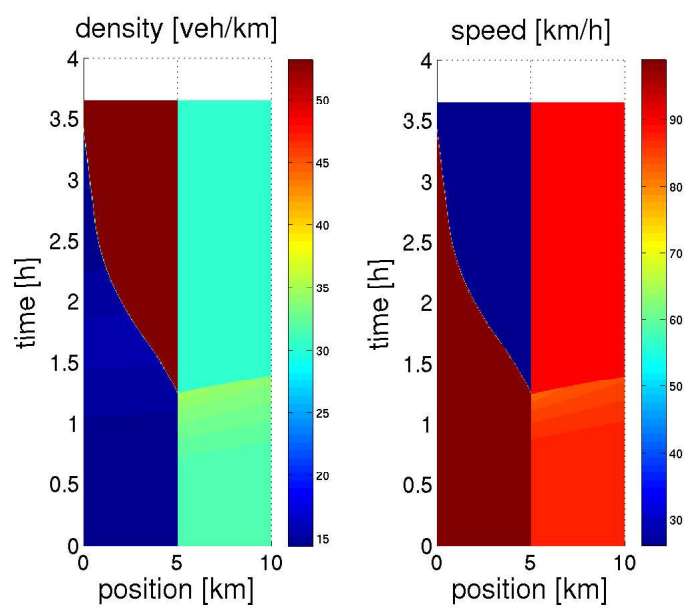

Fig. 6. The evolution of the traffic state in presence of a capacity drop representation.
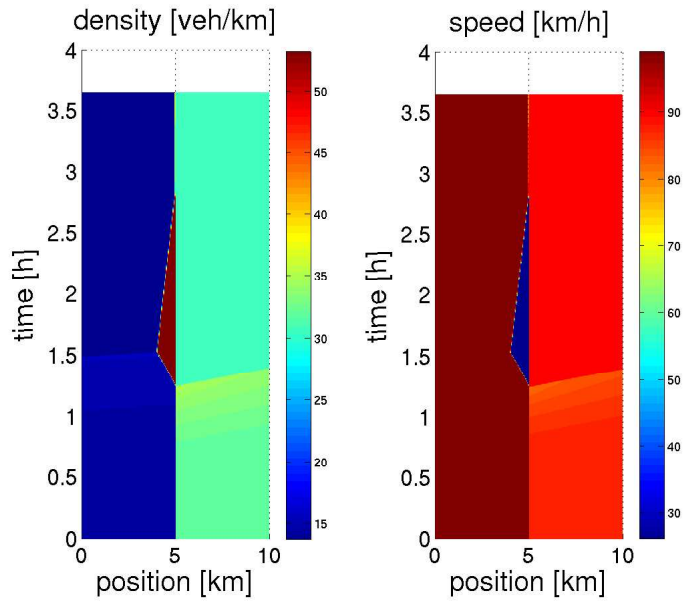

Fig. 7. The evolution of the traffic state in presence of a capacity drop representation and a ramp metering strategy.

\section{CONCLUSIONS}

As we have seen in the previous section, the representation of the capacity drop phenomenon has significant consequences on the system behaviour. Here, the application of a control strategy is meaningful. We can use, for example, some ramp metering strategy (see Papageorgiou (2002)) to maintain the density at the entrance of the junction under a critical threshold. The control will permit to return to the full reception capacity of the outgoing road and make the traffic jam disappear forever as it can be seen in Fig. 7. At first glance, it may seem surprising that the traffic state on road 3 at the end of the simulation is not the same that at the beginning. The reason is that the control strategy, in order to make the traffic jam disappear, had to limit the entering flow. After the traffic jam has disappeared, the vehicles stopped at the entrance of road 1 must still enter into the road so the demand at the end of the simulation is higher than at the beginning.

Without this modification, the control wasn't needed to make the system return to an uncongested state. A correct modelling of the capacity drop phenomenon at the junctions is thus a critical element in the establishment of a traffic state regulation strategy.

If we consider now a more general network with multiple incoming-outgoing roads junction such as in the models Coclite et al. (2004) and Holden and Risebro (1995), the same modification is still possible. We modify the reception capacity of the $j^{\text {th }}$ outgoing road by

$$
R_{j}^{\prime}=\min \left(R_{j}, g\left(\sum_{i} \alpha_{j i} S_{i}\right)\right)
$$

where $\alpha_{j i}$ represent the percentage of drivers from the incoming road $i$ who wish to enter the outgoing road $j$. 


\section{REFERENCES}

Alberto Bressan. Hyperbolic Systems of Conservation Laws - The One-dimensional Cauchy Problem. Oxford University Press, 2000.

M. J. Cassidy and R. L. Bertini. Some traffic features at freeway bottlenecks. Transportation Research Part B, B33:25-42, 1999.

G. M. Coclite, M. Garavello, and B. Piccoli. Traffic flow on a road network. to appear in SIAM J. Math. Anal., 2004.

C.F. Daganzo. The cell transmission model 2: network simulation. Transportation Research $B$, 29B(2):79-93, 1995.

F. L. Hall and K. Agyemang-Duah. Freeway capacity drop and the definition of capacity. Transportation Record, (1320):99-109, 1991.

M. Herty and A. Klar. Modeling, simulation, and optimization of traffic flow networks. SIAM J. Sci. Comput., 25(3):1066-1087, 2003.

H. Holden and N. H. Risebro. A mathematical model of traffic flow on a network of unidirectional roads. Siam J. Math. Anal., 26(4):9991017, 1995.

W.L. Jin and H.M. Zhang. On the ditribution schemes for determining flows through a merge. Transportation Research PartB, (37):521-540, 2003.

JP Lebacque. The godunov scheme and what it means for first order traffic flow models. In Transportation and traffic flow theory, Proceedings of the 13th ISTTT, Pergamon, 1996.

JP Lebacque. Intersection modeling, application to macroscopic network traffic flow models and traffic management. In S. Hoogendoorn and M. Schreckenberg, editors, Proceedings of Traffic and Granular Flow'03. Springer, 2004.

JP Lebacque and MM Khoshyaran. Transportation planning: the state of the art, Macroscopic flow models, pages 119-139. 1998-2002.

M. J. Lighthill and J. B. Whitham. On kinematic waves. i: Flow movement in long rivers. ii: A theory of traffic flow on long crowded roads. Proc. Royal Soc. London Ser. A, (229):281-345, 1955.

HK Lo. A dynamical traffic assignment formulation that encapsulates the cell-transmission model. In Transportation and traffic flow theory, Proceedings of the 14th ISTTT, Pergamon, 1999.

P Nelson and N. Kumar. Point constriction, interface and boundary conditions for the kinematicwave model, trb 2004 annual meeting. 2004.

M. Papageorgiou, J.-M. Blosseville, and H. HadjSalem. Modelling and real-time control of traffic flow on the southern part of Boulevar Périphérique in Paris: Part ii : coordinated onramp metering. Transportation Research Part A, 24A(5):361-370, 1990.
Markos Papageorgiou. Freeway ramp metering : An overview. IEEE transactions on intelligent transportation systems, 3(4):271-281, december 2002.

P. I. Richards. Shock waves on the highway. Oper. Res, (4):42-51, 1956.

N. Elloumi and H. Haj-Salem, METACOR: $A$ Macroscopic Modelling Tool For Urban Corridor, volume I, 135-149, Capri-Italy. Congress Center, June 23-28, 1994. TRISTAN III : TRIennal Symposium on Transportation Analysis. 